\title{
Schematized Access Control for Data Cubes and Trees
}

\author{
Claudio Marxer \\ University of Basel, Switzerland \\ claudio.marxer@unibas.ch
}

\author{
Christian Tschudin \\ University of Basel, Switzerland \\ christian.tschudin@unibas.ch
}

\begin{abstract}
In classic ICN where delivery of named data cannot be guarded, access control is usually implemented by first encrypting the data and secondly by providing the corresponding data encryption keys (DEKs) to authorized users only: Authorized users will obtain DEKs in encrypted form, wrapped with their public key. This approach has three shortcomings which we address in this paper. (a) Key management is tedious if it has to be done on a per-principle basis, (b) access granularity for single documents should be extended to document collections (e.g. namespace sub-trees) and data cubes (sub-elements within data records), (c) there needs to be support for access right propagation across data aggregation and derivation chains.
\end{abstract}

\section{CCS CONCEPTS}

- Security and privacy $\rightarrow$ Access control; Management and querying of encrypted data; $\bullet$ Networks $\rightarrow$ In-network processing;

ACM Reference format:

Claudio Marxer and Christian Tschudin. 2017. Schematized Access Control for Data Cubes and Trees. In Proceedings of ICN '17, Berlin, Germany, September 26-28, 2017, 6 pages.

DOI: $10.1145 / 3125719.3125736$

\section{INTRODUCTION}

Access control for files is a well-known and standard functionality of multi-user operating systems like UNIX: A set of three access rights rwx enables the owner of a directory to control who should be allowed to read, write or list the directory's entries. In an ICN network, typically also coming with a hierarchical namespace, this form of access control is not part of the service contract: Given an arbitrary path into the ICN namespace, the network is supposed to deliver the corresponding data chunk. Access control in ICN can be implemented indirectly, though, by encrypting the data items with DEKs (data encryption keys) and managing the DEK production and dissemination for the authorized principles.

A key question in ICN is whether the consumption side of access control (read access) can be implemented in a fully passive way meaning that there is no need for an online entity or protocol for generating DEKs. This fully passive approach was explored in [6] and works well for small contexts where there is a single layer of consumers and where access granularity and scope can be defined at data production time. However, when primary data should be

Permission to make digital or hard copies of part or all of this work for personal or classroom use is granted without fee provided that copies are not made or distributed for profit or commercial advantage and that copies bear this notice and the full citation on the first page. Copyrights for third-party components of this work must be honored For all other uses, contact the owner/author(s).

ICN '17, Berlin, Germany

(c) 2017 Copyright held by the owner/author(s). 978-1-4503-5122-5/17/09 . $\$ 15.00$ DOI: $10.1145 / 3125719.3125736$ "selectively" accessed according to criteria defined at later times, other venues have to be found. For example, we wish to control access to derived fitness data where primary records are cleansed from their GPS coordinate field. Another example is granting access to a group of consumers which was not known at production time which mandates the generation of new DEKs (otherwise the new group would inherit access to all items which were encrypted with the old DEK used at production time). Our working assumption is a setup where active services, referenced by named functions, are in place for creating new DEKs on-demand and independently of the original data production process.

Security Model. In our security model assets are collections of content objects defined by name patterns (e.g. subtree in hierarchical namespace) but also sub-elements within these data packets. Sub-elements could mean for instance a content object with cleansed fitness data containing only some information of the original record. More technically spoken, selected sub-elements are results of in-network computations as e.g. in Named Function Networking (NFN). Principals, i.e. authorized parties, are organized in an hierarchical tree-like fashion. This means that read access, once granted, also permits all "higher" (ascending) principals to read but does propagate to "lower" (descending) principals. Often, access control is implemented with an online key synchronization protocol or at least with pre-generated and published DEKs. In our case, access control logic enforcement is decentralized and distributed: Consumer and producer share deterministic procedures and certain information which allow authorized parties to generate DEKs in a location and time-independent way. As we show in the following paragraph, assets are annotated with meta-data such that the decentralized access control logic procedures can "reason about" the information-content of a certain asset and match against a capability list in which the access-control policies are materialized.

System Architecture. Figure 1 lays out the component of our access control system. Note that this figure deliberately avoids showing any packet-level exchanges or "network clouds" and attachment points. Instead, it shows pure data structure relations and names each pointing into different names spaces: An arrow stands for a name that references documents or processes. The core and center of this figure is the time and location-independent derivation procedure of DEKs: A producer uses it for all data and derived computation results that will be published in the ICN, while the consumers also needs to derive the same keys in order to decrypt the data. Decentralized access control enforcement is implemented by the pentagon-shaped access control logic enabling - or not producers and consumers to derive DEKs. The actions of this access control logic are driven by a series of metadata documents capturing the access policies. The capability document (CAP) links assets (shown to the left) with principles (shown to the right) and is the central document controlling the DEK generation system. 
Technically, the CAP states which principals are authorized to request for which asset certain "seed" information to perform the DEK generation procedure.

Section 2 recapitulates basic concepts of ICN and NFN. Section 3 introduces metadata to express data access granularity for content matching certain name-based patterns but also finer-grained inrecord fields and value classes, leading to a data cube view. The subsequent section shows how this data organization maps to different query possibilities on which access control should be imposed in a decentralized way (Section 4). We conclude the paper with an example in Section 5.

\section{BACKGROUND}

In this section we introduce Named-Data Networking and the Named-Function extension. Furthermore, access control challenges and techniques in this context are described.

\subsection{Named-Data and -Function Networking}

In Information Centric Networking (ICN) content chunks are treated as the base object. Content is addressed by names which have no reference to any servers, enabling the ICN network to reorganize content distribution but also putting the ICN in charge of finding the content based on the name alone. Two popular flavors of ICN have been CCNx [2] and NDN [1] (and more recently CICN) which are based on hierarchical names: Content names are paths in the data namespace e.g. dat:/this/name/has/five/components.txt. In order to fetch content, an Interest packet is sent to the network and a Data packet is returned on success. Name and content are bound via signatures, always part of a content chunk, such that the consumer (and potentially also forwarding elements) can verify the authenticity of received content.

Named Function Networking (NFN) augments ICN with in-network content derivation capabilities [4]: Names are expressions which include data and function names. E.g., the consumer can request the result of function wordcount () applied to the content object poem. txt:

fct:/unixstd/wordcount ( dat:/a/nice/poem.txt )

Such an expression instructs the network to either get hold of the corresponding result (if it already exists), or to let the network orchestrate its computation. In the latter case, NFN-enabled network nodes decompose (=reduce) an expression and recursively work through the sub-expressions. Once a (sub-) result is obtained, it is turned into an object whose name is exactly the (sub-) expression.

In this paper we deliberately keep the namespaces (dat: and fct:) distinct although in some of our prototypes we have found ways to map NFN names onto the ICN namespace of NDN.

\subsection{Access Control via Data Encryption Keys}

Restricting access to some content (regardless whether stemming from the dat: or fct: namespace) is achieved through Data Encryption Keys (DEK). Although a content chunk can be fetched by anybody, only principles possessing the corresponding DEK can access the clear text. Using a symmetric DEK key and wrapping it once for each authorized principle with its public key permits to publish a new content item such that all principles can read it by decrypting with the unwrapped DEK. By linking the name of the DEKs (through conventions) to the name of the content to be protected, we obtain "named-based access control" (NAC) as it was described in [6].

In a NFN context, an additional complexity is introduced by not knowing in advance which node will produce a (sub-) result and what access policies -linked to the input parameters- have to be obeyed. In order to decouple data generation, access control and result consumption in space and time we need to derive DEKs based on a function's shape and input parameters in a deterministic way, both for the producing but also for the consuming entity. This is shown in Fig. 1 by the two DEK derivation arrows for the prod and cons triangles. One of the consequences we elaborate on in this paper is that unlike with NAC, key names cannot be easily attached to the data namespace anymore.

\section{DATA ORGANIZATION}

The classic tree-shaped organization of content leads to the problem that access patterns influence the shape of the tree. For example, if fitness data is queried by event first, it will be organized as dat: . . /NYCmarathon/2016/record1234 although one could also organize it first according to time as done with a name like dat: ... /2016/NYCmarathon/record1234. Moreover, a single record will have several attributes (series of GPS location and timestamps, comment fields), which in general will and cannot all be made visible at the ICN name level. NFN permits to overcome this problem by introducing (field) extraction functions. In this section we elaborate on organizing data as a logical data cube. We argue that access levels are useful to express degrees of precision on which access decisions should be based. Moreover, we point out the need for metadata documents that express such data organization details.

\subsection{Access Granularity}

"Records" are the classic way of storing content in a database. It also naturally lends itself to be used as the "publishing unit" for e.g. fitness data records: Multiple field values (heartbeat, GPS coordinates) are grouped and annotated with a timestamp, and would be published as a single piece of ICN content having its own name. A second observation is that the fields are often drawn from a discrete set of values and can be mapped to classes. For example, low/med/high temperature, or exact/approx/relocated coordinates. Both cases (multiple fields, multiple value classes) provide access granularity based on which we wish to make access control decisions. In the following, we elaborate a use case with time-locationcomment records and introduce the geometric interpretation in form of a data cube.

Example: Time-Location-Comment Tracks. Figure 2.(a) shows a JSON-encoded sample of a primary time-location-comment record. The key "location" maps to an array of longitude-latitude pairs (waypoints) while "time" maps to an array of timestamps reflecting the capturing time of each waypoint. The key "comment" maps to a string in which the record might be described by the producer.

For the general case it is suitable to treat time and location series as well as the comment value as fields. This allows to address either of these three fields separately or exclude one of them. For each field, the highest accuracy level is constituted by the captured data ("primary") while from the lowest level no information can be 




Figure 1: Big picture showing the relation of data structures, processes and namespaces.

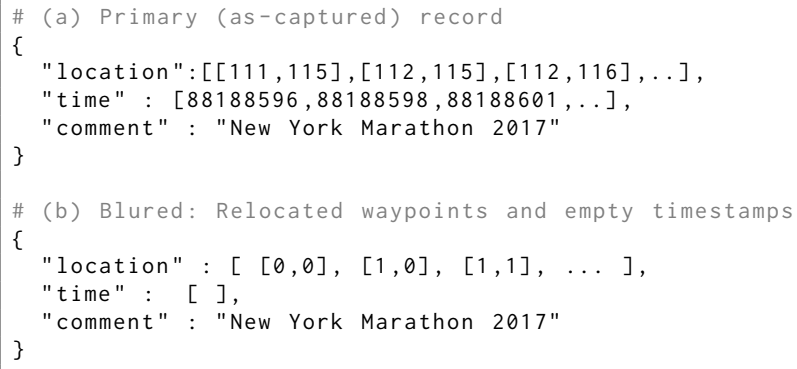

Figure 2: Two variants of a record that contains waypoint coordinates as well as capturing times and a comment.

learned ("empty"). For the time and location section we propose to have another intermediate accuracy level which sets the first timestamp/waypoint to a canonical starting value but preserves the temporal/spacial deltas from point to point. This means, a relative waypoint series reveals no real-world whereabouts but contains still sufficient information to compute for example the length of the represented track. Variant (a) shows the primary (as-captured) record while (b) shows the same record with blured waypoints ("relative") and timestamps ("empty").

(Hyper) Cube Representation. In-record access-granularity determined through dissecting and bluring is more intuitively captured by a (hyper) cube as depicted in Figure 3 . The fields of the content constitute the dimensions of the cube and the according accuracy levels define each dimension's spectrum. Since we proposed to decompose time-location-comment records into three fields, the dimensions of the depicted cube are time, location and comment. The values on the location dimension correspond to the accuracy levels "primary" (L), "relative" (1) and "empty" $(\epsilon)$. The same holds for the time dimension where the values are "primary" $(\mathrm{T})$, "relative" (t) and "empty" $(\epsilon)$. Last, the comment dimension is defined for "primary" (c) and "empty" $(\epsilon)$.

In our example, the cube consists of 18 subcubes from which each is associated with a different combination of accuracy levels. For instance, subcube (1) describes a full record (no blured information, "primary" on all dimensions). Different subcube (2), where all time and location information is blured but only the comment is preserved. We identify subcube (1) with LTc and subcube (2) with c. In general, a subcube identifier concatenates the values from each dimension whereby permutations (e.g. LTc, TLc, LCT) are equal and $\epsilon$ can be omitted.

\subsection{Document Types}

A cube captures the access granularity within a certain record type. Together with meta-data (e.g. measuring units), this information is compiled into a Document Type Definition (DTD) and made available as a content object. Figure 4 shows the DTD for records of type timelocation-comment. In line 2, the dimensions of the according cube are defined. Lines 3 to 7 outline the accuracy levels of all dimensions. In line 8 it is indicated that meta-data such as measurement units for geo-points and timestamps should be stated in the DTD. Like other data exchange formats, DTDs can be defined by standardization bodies. For instance, the depicted DTD would be available as dat:/ieft/type/time-location-comment.dtd A DTD itself 


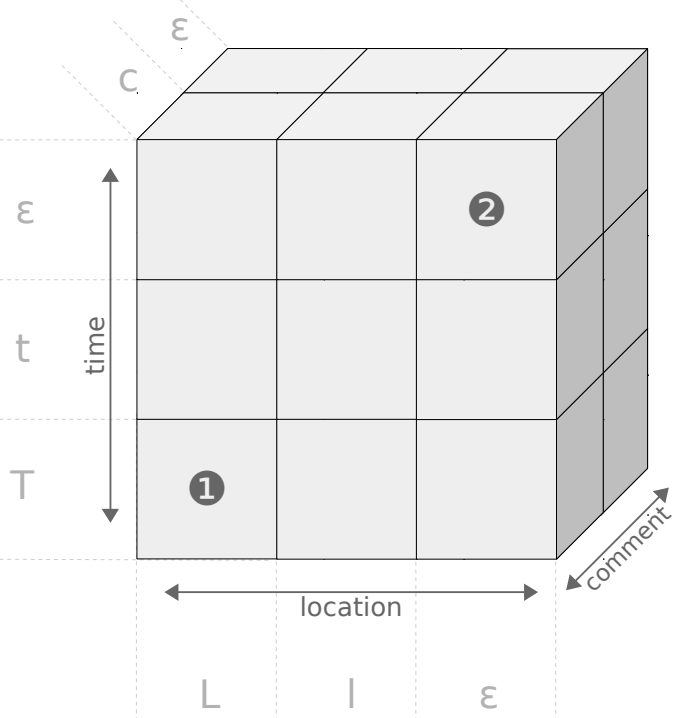

Figure 3: (Hyper) Cube: Capturing in-record accessgranularity for time-location-comment records.

just states the existence of certain dimensions and levels in a cube. For the mechanics of our access control system there is no technical need to provide a semantical description of these. If other parts of an application need such information, this could be included in DTDs or might be placed in another metadata document.

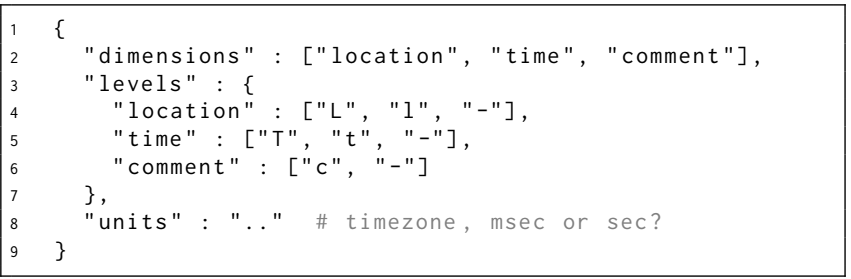

Figure 4: Document Type Definition (DTD) for timelocation-comment records.

\subsection{Linking Records and Types}

Data publishers must specify the document type of each published record. With Document Type Schemas (DTS) we propose a mapping based on name patterns (grep style in our case). Figure 5 depicts a DTS which consists of three rules. The first rule states that direct children from dat:/joe/workout name records of type time-location-comment.dtd. As these examples show, document types can be assigned to entire subtrees but more selective pattern are feasible as well. By convention, a DTS states implicitly that all captured records are primary (i.e. come with highest accuracy level on all dimensions). For instance, if there exists a content object dat: / joe/workout/record1234, it is of type time-locationcomment.dtd) and the record contains primary data without any blured parts (described by subcube LTc).



Figure 5: Document Type Schema (DTS) for collection dat:/joe/workout (and a generic rule for ASCII text).

\subsection{Linking NFN Queries and Subcubes}

Accessing fields of a record can be easily expressed via an NFN function e.g. TakeComment (). A Derived Data Schema (DDS) defines associations of data extraction result with subcubes. Figure 6 shows an example containing filters introduced for time-location-comment records. Each line constitutes one rule which states the effect of one filter or extractor. The left side of a rule consists of the function's name and a placeholder for arguments (\#). The argument \# stands either for a primary record (e.g. dat: /joe/workout/record1234) or the previous step in a filtering chain (recursively captured by another other rule). The right side of a rule states the effect of the function on its input. The placeholder @N stands for the subcube of $\# \mathrm{~N}$ and the $\backslash$ operator means that all information higher or equal to the precision from right-hand side is removed.

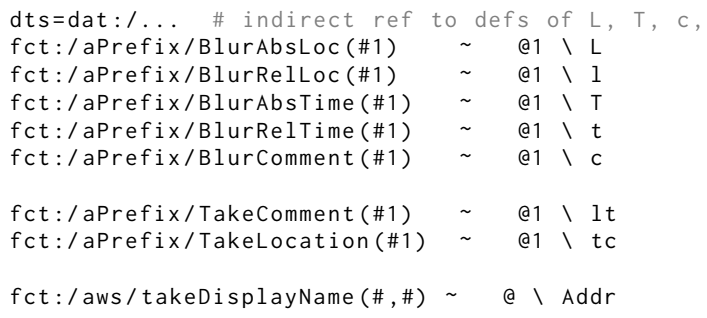

Figure 6: Derived Data Schema (DDS).

According to this DDS and the introduced DTS, the query

fct: / aPrefx/TakeComment (dat:/ joe/workout/record1234)

maps to the subcube $c$ because the DDS states that TakeComment () blures all time and location information but preserves the comment section. According to the DTS, the input is a primary record, hence associated with subcube LTc. Thus, in this concrete case, the extractor function performs LTc $\backslash 1 t$, which results in subcube $c$. The last line specifies a function pattern with two-parameters (database reference and persId) and suppresses the address field of its records.

\section{ACCESS CONTROL}

In this section we show how to restrict access (i.e., provide DEKs) to authorized parties only. A principal can be an individual consumer, a group or a NFN processing node that performs filtering tasks. For our solution these distinctions do not matter since each is associated with an own public-private key pair. Our approach is also based on schemata as in [5] but uses them to control access, not for validating the trustworthiness of keys. The DEK for a certain 
result must be the same anywhere at any time. This is achieved with a deterministic derivation procedure as proposed in [3].

\subsection{Security Contexts and Key Trees}

A frequent pattern is that a group of principals should enjoy the same privileges. We make this group concept explicit by introducing security contexts. A single (usually symmetric) DEK is assigned to a security context such that all its members share read privilege. Moreover, we organize security contexts in a hierarchical fashion in order to automatically organize supervisor privileges: Because a parent context has access to the credentials of all its children security contexts, it suffices to encrypt for a single group level which will automatically grant access to all contexts up to the root of such a "key tree" (see upper right corner of Fig. 1). We map this hierarchy to a path where the first component of a key name is the owner of the key tree.

key: / AAPL/Cupertino/SecDept/Jane. Roe

key : /RSAPubJohnDoe/email/providerA

For example, an employee in Apple's security department has a personal security context for her daily work. In an escrow-like fashion, the department (and thus also Apple's master key) has access to all her encrypted data, but also to new security contexts she will create under her key-tree-prefix. The same applies to John Doe's key tree where he can organize his private security contexts topic-wise and invite, for example, an email provider to share the providerA context, without granting access to John's other security contexts.

Technically, a security context requires an "encrypted broadcast" primitive allowing its members, and only them, to securely exchange data. We implement this by letting the parent context create a public-key pair used for controlling member records and one or more DEKs to be used for data shared within a security context. Members are added by encrypting the DEKs with each member's public key. A new child context can be created by any group member by broadcasting the new public-key pair to all group members (which will extend to all parent contexts automatically) Note that the action of "broadcasting" simply means to publish the new key pair encrypted with the group's management DEK.

\subsection{Granting Access to Principals}

The capability list $(C A P)$ is the central metadata that links patterns of derived data with principles. It does so by assigning access right names (as defined in a DDS) to principles (as defined in a key tree). The following listing shows an example capability list with three principals.

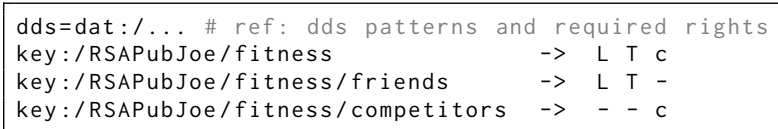

The principal identified with .../fitness is allowed to read full records in the collection because the list includes the highestpossible precision on all dimensions. Another principal identified with .. /competitors can just read comments since the capability list states that time and location dimension are entirely locked down (-).

\subsection{Enforcing Access Rights}

At run-time, a principle's access rights are "matched" with the minimum rights required to access some content (specified through Derived Data Schema). Matching in this content means that a DEK can be derived only when sufficient rights have been granted. The DEK derivation procedure itself must be authorized to perform such a computation, which is enforced by the same mechanism (by making the executing principle a member of the respective security context). This computation could, if the owner trusts the provider, then be part of a computation chain fully executed in the cloud yet controlled by our access control system.

The code skeleton depicted in Figure 7 shows the access control logic. A consumer or producer that belongs to a security context secCtxName will access the DEK for a given expression expr by computing it with the deriveDEK() function. This value, which can be cached, is used in the access() function to decrypt the requested result.

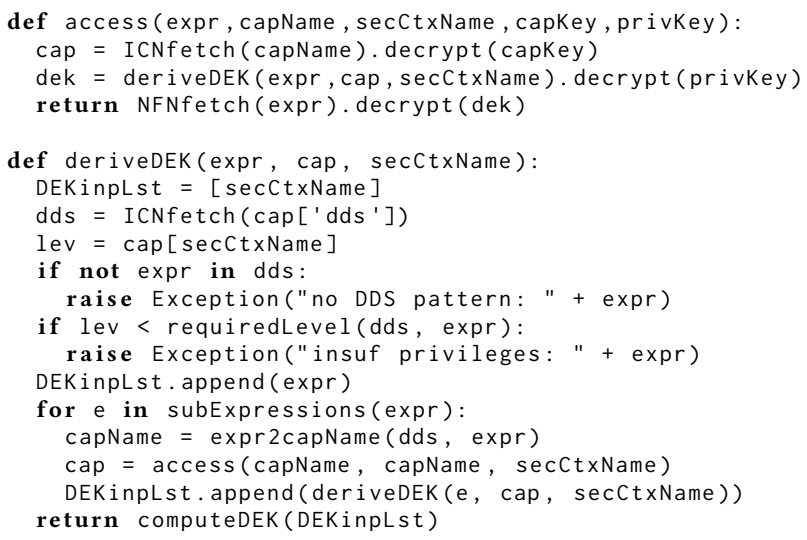

Figure 7: Pseudocode: Access Control Logic

The capKey parameter to access() is the decryption key for the CAP document and points out that the capabilities file is itself subject to access restrictions. The access () procedure is executed in a secure context i.e. both the producer or consumer device. The role of the deriveDEK() is to deterministically compute a symmetric key, both by the producer and its consumers, that protects a result. This is done by collecting elements unique to the given expression and by deriving from them through repeated hashing a final DEK. A NFN node that has to compute an intermediate result e.g. fusing two data sources and returning a combined result will check the requester's credentials for matching entries in the CAP and DDS and also verify access to sub-expressions recursively.

\section{EXAMPLE}

The example given in this section involves Joe, Ann and Ron. Joe is maintaining a list of work buddies indexed by persID numbers that refer to a person database: Ann and Ron should be permitted to access the display name of Joe's work buddies but should not have access to the corresponding addresses stored in the person records. We also introduce the storage and compute providers that Joe is 
trusting. Overall, the key tree rooted in Joe's public key would look like this:



Figure 8: Joe's key tree. Each node represents a security context. Comments show the members of the leaf node.

The pretty-printed NFN name of "the list of display names of Joe's work buddies" would look like this:

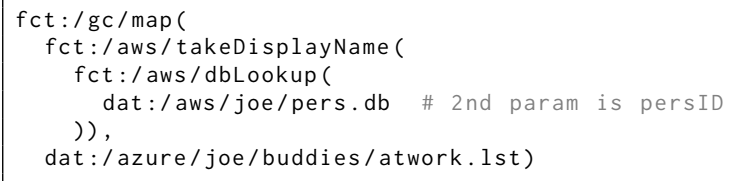

The map function, executed by Google's cloud, applies the extraction process that is executed by the Amazon Web Services and combines data coming from Microsoft's Azure cloud. To this end, Joe grants read access for the buddy list to GC via the Azure2GC security context. AWS stores and has read access to the person DB: It makes the extracted display names available to GC via the AWS2GC security context, also controlled by Joe. Ann and Ron, finally, have access to the result of GC, but not to any intermediate results nor to the primary data sources. Joe's key, sitting at the top of the key tree, provides access to all final as well as intermediate results. Note that a security context has its own key: Although AWS's public key will be part of many security contexts worldwide, Joe's security context instance is different from Jim's.

CAP files must in place where results cross trust domains. For example, AWS must be able to read full person records and extract single fields, while GC only has access to the extracted display name. Also, corresponding derivation schemata must be in place. We only show the required entries for the last stage of the map result (and combine the content of the different schemata into one listing):

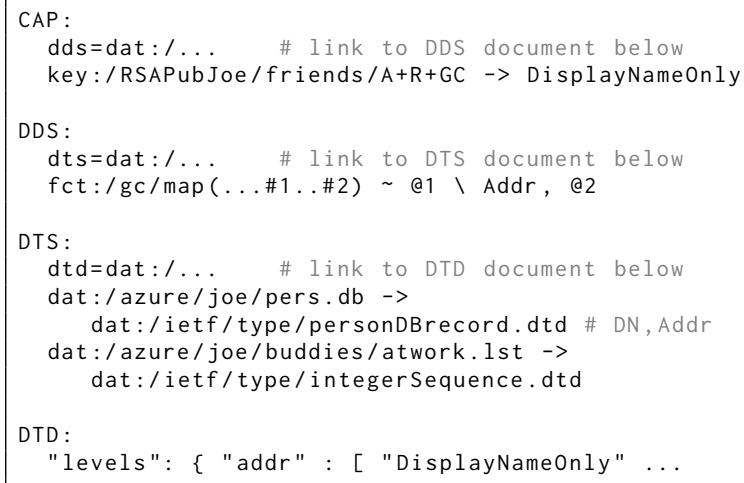

\section{KEY INSIGHTS AND CONCLUSIONS}

This paper studies how names for data types, functions, data chunks and security groups can be organized for the benefit of access control. This is a challenging task because unlike in an operating system context there is no single trusted execution place which would check and enforce access rights. The role of access logic is to derive the data encryption keys for authorized principles based solely on documents stored in the ICN network - we identified four different schemata to this end. The subjects of access control, the principles, are organized in groups called security contexts, which is another contribution of this paper.

A key insight is that typing and annotating content plays a central role in orchestrating access control. First, we have different name types (into the ICN's data space, into NFN's function space, into the key space and regarding access granularity). Previous work in the ICN community had assumed that a single name space would be sufficient into which all these concerns could be mapped, and consequently came up with conventions how, for example, a data encryption key would have to be named that gave access to some data item. We deliberately separated these namespaces, which we think is essential for handling access and processing chains. The second, obvious reason for typing is that metadata is anyway mandatory e.g., knowing the measurement units in an IoT, M2M or T2T scenario.

When providing all the meta-data and access-control relavant information on the network layer and further using an "in-network computation layer" to address sub-elements within data packets, one could argue that access-control is merged at least partially with the network layer. It is an insight that this is necessary if access-controlled content should be derived on-demand and in an location-independent way.

The overall machinery for access control looks "involved" but we expect that most of the complexity can be hidden from the programmer behind libraries and schemata generators.

\section{ACKNOWLEDGMENT}

We thank Christoper Wood (UC Irvine) for discussions on an early version of this paper.

\section{REFERENCES}

[1] Alexander Afanasyev, Junxiao Shi, Beichuan Zhang, Lixia Zhang, Ilya Moiseenko, Yingdi Yu, Wentao Shang, Yanbiao Li, Spyridon Mastorakis, Yi Huang, Jerald Paul Abraham, Eric Newberry, Steve DiBenedetto, Chengyu Fan, Christos Papadopoulos, Davide Pesavento, Giulio Grassi, Giovanni Pau, Hang Zhang, Tian Song, Haowei Yuan, Hila Ben Abraham, Patrick Crowley, Syed Obaid Amin, Vince Lehman, Muktadir Chowdhury, and Lan Wang. 2016. NFD Developer's Guide. Technical Report NDN-0021, Revision 7. NDN. http://named-data.net/ techreports.html

[2] Van Jacobson, Diana K. Smetters, James D. Thornton, Michael F. Plass, Nicholas H. Briggs, and Rebecca L. Braynard. Networking Named Content. In Proceedings 5th International Conference on Emerging Networking Experiments and Technologies (CoNEXT'09).

[3] Claudio Marxer, Christopher Scherb, and Christian Tschudin. 2016. AccessControlled In-Network Processing of Named Data. In Proceedings of the 3rd ACM Conference on Information-Centric Networking (ACM-ICN '16). ACM, New York, NY, USA, 77-82. DOI : https://doi.org/10.1145/2984356.2984366

[4] Manolis Sifalakis, Basil Kohler, Christopher Scherb, and Christian Tschudin. 2014. An Information Centric Network for Computing the Distribution of Computations. In Proceedings of the 1st ACM Conference on Information-Centric Networking (ACM-ICN '14). ACM, New York, NY, USA, 137-146. DOI : https: //doi.org/10.1145/2660129.2660150

[5] Yingdi Yu, Alexander Afanasyev, David Clark, Van Jacobson, Lixia Zhang, and others. 2015. Schematizing trust in named data networking. In Proceedings of the 2nd International Conference on Information-Centric Networking. ACM, 177-186.

[6] Yingdi Yu, Alexander Afanasyev, and Lixia Zhang. 2016. Name-Based Access Control. Technical Report. Internet Research Lab, UCLA. 